\title{
A multi-time-step noise reduction method for measuring velocity statistics from Particle Tracking Velocimetry
}

\author{
Nathanaël Machicoane ${ }^{1}$ \& Miguel López-Caballero ${ }^{2}$ \& \\ Mickael Bourgoin ${ }^{3}$ \& Alberto Aliseda ${ }^{1}$ \& Romain Volk ${ }^{2}$ \\ ${ }^{1}$ University of Washington - Department of Mechanical Engineering, Seattle, WA, \\ USA \\ ${ }^{2}$ Univ Lyon, Ens de Lyon, Univ Claude Bernard, CNRS, Laboratoire de \\ Physique, F-69342 Lyon, France \\ ${ }^{3}$ LEGI, Université de Grenoble/G-INP/UJF/CRNS, BP53 Grenoble, 38041 cedex \\ 9, France \\ January 2017
}

\begin{abstract}
We present a method to improve the accuracy of velocity measurements for fluid flow or particles immersed in it, based on a multi-timestep approach that allows for cancellation of noise in the velocity measurements. Improved velocity statistics, a critical element in turbulent flow measurements, can be computed from the combination of the velocity moments computed using standard Particle Tracking Velocimetry or Particle Image Velocimetry techniques for data sets that have been collected over different values of time intervals between images. This method produces Eulerian velocity fields and Lagrangian velocity statistics with much lower noise levels compared to standard PIV or PTV measurements, without the need of filtering and/or windowing. Particle displacement between two frames are computed for multiple different timestep values between frames in a canonical experiment of homogeneous isotropic turbulence. The second order velocity structure function of the flow is computed with the new method, and compared to results from traditional measurement techniques in the literature. Increased accuracy is also demonstrated by comparing the dissipation rate of turbulent kinetic energy measured from this function against previously validated measurements.
\end{abstract}

Submitted to: Meas. Sci. Technol. 


\section{Introduction}

Flow velocity measurements, based on the analysis of the motion of particles imaged with digital cameras, have become the most commonly-used metrology technique in contemporary fluid mechanics research [1]. Particle Image Velocimetry (PIV) and Particle Tracking Velocimetry (PTV) are two widely used methods that enable the characterization of a flow from the Eulerian (PIV) or Lagrangian (PTV) point of view.

Particle Tracking Velocimetry is a versatile measurement technique to probe complex fluid mechanics problems, when the particles are fluid tracers, and particle-fluid interactions, when the particles have inertia and do not follow the flow $[2,3,4,5]$. Recently, multi-camera approaches and wider illumination regions have become widely available for particle tracking enabling the measurement of the three components of the velocity in a relatively thin volume (3C-2D) and fully three dimensional measurements of the three components of the velocity at a prismatic volume in the flow (3C-3D). Two frequent realizations of this method in the laboratory are based on taking a pair of images, using double exposure cameras, typical of PIV, at very short time separation followed by a large time interval, or collecting a long sequence of images closely and equally separated in time, using high speed cameras. The first technique provides a single vector per particle in a pair of consecutive images, with subsequent velocity measurements in other image pairs being uncorrelated from this. The high speed image sequence, on the contrary, provides the opportunity to track the same particle over multiple images and provides several correlated velocity measurements, at different locations but along the same particle trajectory.

Noise reduction in velocity measurements based on the displacement of particles from images of light scattered have been developed and tested extensively. From tracking detection from multiple sequential images $[6,7,8,9]$ to improved centroid detection [10], error estimation [11] and camera calibration [12], the advantages of noise-reduction on imaging or particle detection for improved accuracy of velocimetry have been demonstrated. This note takes a completely different tack on noisereduction. The novel method presented here removes noise not from the individual image or particle center detection, but globally in the calculation of velocity statistics, by using the redundant information in measurements taken from image sequences collected at multiple time steps. The presentation uses as an example the 
computation of Eulerian velocity statistics by spatial binning in order to determine flow features and turbulence characteristics (such as dissipation).

\section{Background on particle location measurement noise}

Experimental noise in the position of particles in an image is particularly critical for measurements of the velocity or acceleration fields, where differentiation is needed. PTV introduces noise both from the light scattering and imaging physics, and from the tracking/correlation mathematical processing of those positions $[1,13]$.

In PTV, particle positions are determined, including measurement noise, by detecting the centroid of each region of the camera sensor that records light intensity above a certain threshold in contiguous pixels. While the uncertainty in the detection of the centroid as the true location of the particle center does not represent a significant problem in the computation of particle location statistics, the noise in the particle location becomes a severe limitation when using these measurements to calculate velocity or acceleration (first and second derivatives, respectively). The need to filter the particle positions prior to any differentiation has long been recognized $[4,14,15,16]$. Multiple filter parameters, for example filter length, have to be chosen or adjusted to minimize the propagation and amplification of noise in the process of taking the derivative of the measurements. How the derived quantities depend on the filtering can be studied to provide confidence in the data, even leading to information on the expected value of some moments of the measurement distribution (the rms value of particle acceleration in $[14,15])$. Filtering, however, generally removes information, erasing events (changes in particle trajectories) with time scales shorter than the filter length.

If the particle position is $\mathbf{X}(t)$ and the Lagrangian displacement field is $\Delta \mathbf{X}=$ $\mathbf{X}(t+\Delta t)-\mathbf{X}(t)$, noise $\mathbf{b}$ in the detected position $\widetilde{\mathbf{X}}$ biases the statistics computed using a naive definition of the velocity $\Delta \mathrm{X} / \Delta t$. The method described here computes noiseless nth moments and nth order structure functions of the velocity field, assuming white noise: $\langle\mathbf{b}(t) \mathbf{b}(t+\Delta t)\rangle_{t}=\left\langle\mathbf{b}^{2}\right\rangle_{t} \delta(\Delta t),\langle\mathbf{b} \Delta \widetilde{\mathbf{X}}\rangle_{t}=\langle\mathbf{b}\rangle_{t}\langle\Delta \widetilde{\mathbf{X}}\rangle_{t}=0$ where \langle\rangle$_{t}$ is an average over time.

The displacement field being $\Delta \mathbf{X}=\Delta \widetilde{\mathbf{X}}+\Delta \mathbf{b}$, its second moment can be expressed as:

$$
\left\langle(\Delta \mathbf{X})^{2}\right\rangle_{t}=\left\langle\widetilde{\mathbf{v}}^{2}\right\rangle_{t} \Delta t^{2}+2\left\langle\mathbf{b}^{2}\right\rangle_{t}+\langle\widetilde{\mathbf{a}} \widetilde{\mathbf{v}}\rangle_{t} \Delta t^{3}+o\left(\Delta t^{3}\right)
$$




\section{Noise-removal algorithm}

The binned Lagrangian displacement (subtracted of the time-averaged displacement in each bin, free of white noise as shown above), gives an Eulerian field of fluctuating displacement to compute the velocity structures in time or space.

To remove the noise from the velocity statistics, $\langle\Delta \mathbf{X}\rangle_{t}$ and $\left\langle(\Delta \mathbf{X})^{2}\right\rangle_{t}$ are calculated for multiple experimental sets where images of the particles in the flow are collected at increasing values of $\Delta t$. When the evolution of $\left\langle(\Delta \mathbf{X})^{2}\right\rangle_{t}$ with $\Delta t$ is fitted by a polynomial of the form $c_{1} \Delta t^{2}+c_{2}$, the leading coefficient is the velocity variance $\left\langle\widetilde{\mathbf{v}}^{2}\right\rangle_{t}$. The third order correction is negligible because dimensional analysis gives $\left\langle\widetilde{\mathbf{v}}^{2}\right\rangle_{t} /\langle\widetilde{\mathbf{a}} \widetilde{\mathbf{v}}\rangle_{t} \tau_{\eta} \sim R e_{\lambda}$, where $\tau_{\eta}=\sqrt{\nu / \varepsilon}$ is the dissipative time and $R e_{\lambda}$ is the Reynolds number at the Taylor length-scale. In turbulent flows, $\langle\widetilde{\mathbf{a}} \widetilde{\mathbf{v}}\rangle_{t}$ is well approximated by the dissipation rate $\varepsilon$. Taking $\Delta t$ smaller than the dissipative time ensures that the displacement field variance is well approximated. The advantage of this method is that it uses all the measurements taken at different values of $\Delta t$ without having to choose any particular $\Delta t$, optimized according to some metric. This method can be extended to higher order moments of the displacement field, as well as to recover increment statistics, for example the longitudinal second order structure function of the velocity $\left(\widetilde{S_{2}}=\left\langle\left[(\widetilde{\mathbf{v}}(\mathbf{X}+\mathbf{r})-\widetilde{\mathbf{v}}(\mathbf{X})) \cdot \mathbf{e}_{\mathbf{r}}\right]^{2}\right\rangle\right.$ (where $\cdot$ is a scalar product and \langle\rangle an ensemble average), with $\left.\mathbf{e}_{\mathbf{r}}=\Delta \mathbf{X} /|\Delta \mathbf{X}|\right)$, by fitting the evolution of $\left\langle\left[(\Delta \mathbf{X}(\mathbf{X}+\mathbf{r})-\Delta \mathbf{X}(\mathbf{X})) \cdot \mathbf{e}_{\mathbf{r}}\right]^{2}\right\rangle$ with a polynomial $\widetilde{S}_{2}(|\mathbf{r}|) \Delta t^{2}+c_{2}$ :

$$
\begin{aligned}
& \left\langle\left[(\Delta \mathbf{X}(\mathbf{X}+\mathbf{r})-\Delta \mathbf{X}(\mathbf{X})) \cdot \mathbf{e}_{\mathbf{r}}\right]^{2}\right\rangle=\left\langle\left[(\Delta \mathbf{b}(\mathbf{X}+\mathbf{r})-\Delta \mathbf{b}(\mathbf{X})) \cdot \mathbf{e}_{\mathbf{r}}\right]^{2}\right\rangle+ \\
& \left\langle\left[(\widetilde{\mathbf{v}}(\mathbf{X}+\mathbf{r})-\widetilde{\mathbf{v}}(\mathbf{X})) \cdot \mathbf{e}_{\mathbf{r}}\right]^{2}\right\rangle \Delta t^{2}+\left\langle\left[(\widetilde{\mathbf{v}}(\mathbf{X}+\mathbf{r})-\widetilde{\mathbf{v}}(\mathbf{X})) \cdot \mathbf{e}_{\mathbf{r}}\right]\left[(\widetilde{\mathbf{a}}(\mathbf{X}+\mathbf{r})-\widetilde{\mathbf{a}}(\mathbf{X})) \cdot \mathbf{e}_{\mathbf{r}}\right]\right\rangle \Delta t^{3}+o\left(\Delta t^{3}\right)
\end{aligned}
$$

Note that the structure function computation does not require the conversion of displacements to Eulerian coordinates, but rather to bin the inter-particle distance $|\mathbf{r}|$. This means that measuring structure functions is possible at arbitrarily small separations $|\mathbf{r}|$, without any requirements on the Eulerian spatial binning. This method requires only a statistical convergence in the number of particles $N$ at a certain range of inter-particle distance (a number that goes with $N^{2}$ ). This represents a significant advantage over methods for structure function computation that carry an associated increase in measurement noise at small separations $|\mathbf{r}|$.

The second order moment of the velocity fluctuations and second order structure function are presented here as examples of what the expansion of statistical moments, 
combined with data collected at different $\Delta t$ can achieve. Higher order moments for the velocity fluctuations and higher order structure functions can be easily computed by this method with reduced noise, although they will contain residual noise from the computation of lower order moments ( $o\left(\Delta t^{3}\right)$ terms above).

\section{Results}

Particle displacements measured in a homogeneous, isotropic turbulence experiment $[17,18]$ are used to demonstrate the validity and accuracy of the method. Two CMOS cameras with a resolution of $2048 \times 1088$ pixels were used in a stereoscopic arrangement. Images were collected in double-frame mode, separated by a time-step $\Delta t$ from $0.05 \tau_{\eta}$ to $0.2 \tau_{\eta}$. Alternatively, a very fast acquisition/illumination rate using high speed camera and $\mathrm{kHz}$ pulsed lasers allows to collect a single image sequence and then take a variable $\Delta t$ in the analysis by skipping an increasing number of images in the sequence. Measurements were obtained in a volume of $10 \times 10 \times 1 \mathrm{~cm}^{3}$ using a Nd:YAG laser. For each experiment, approximately 10000 pairs of image sets per time-step (each set providing the 3D position of several hundred particles in the flow) were collected to ensure statistical convergence.

The different results for the longitudinal second order structure function of $\Delta \mathbf{X}$ (figure 1a) at different time steps, $\Delta_{t}$, show in their shape a strong dependency on how the noise affects the signal for different values of $\Delta t$. The displacement correlation plotted at fixed separations (five different values) are all quadratic in $\Delta t$ (figure $1 \mathrm{~b}$ ), showing this approximation is robust for different levels of measurement noise. The trend $c_{1} \Delta t^{2}+c_{2}$ from equation 2 is followed at different values of the separation $|\mathbf{r}|$, with the positive values of $c_{2}$ being proportional to the variance of the noise (Eqn. 1). The quadratic coefficient $c_{1}$ is the second order function of the velocity with the noise removed. The presence of the inertial range is highlighted by the 2/3 slope in Figure 2a, over approximatively one decade, in good agreement with Kolmogorov's prediction for the second order structure function in homogeneous isotropic turbulence $\left(\widetilde{S_{2}} \sim \varepsilon^{2 / 3}|\mathbf{r}|^{2 / 3}\right)$ [19]. Turbulence variables extracted from velocity measurements would be subject to a significant level of uncertainty and inaccuracy (seen in Figure 1(a)) if the noise were not removed by the method proposed here.

Figure $2 \mathrm{~b}$ shows the estimation of the dissipation rate of turbulent kinetic energy,

$\varepsilon_{r}=\widetilde{S}_{2}^{3 / 2} /|\mathbf{r}|$ for three different Reynolds numbers studied in this experimental 

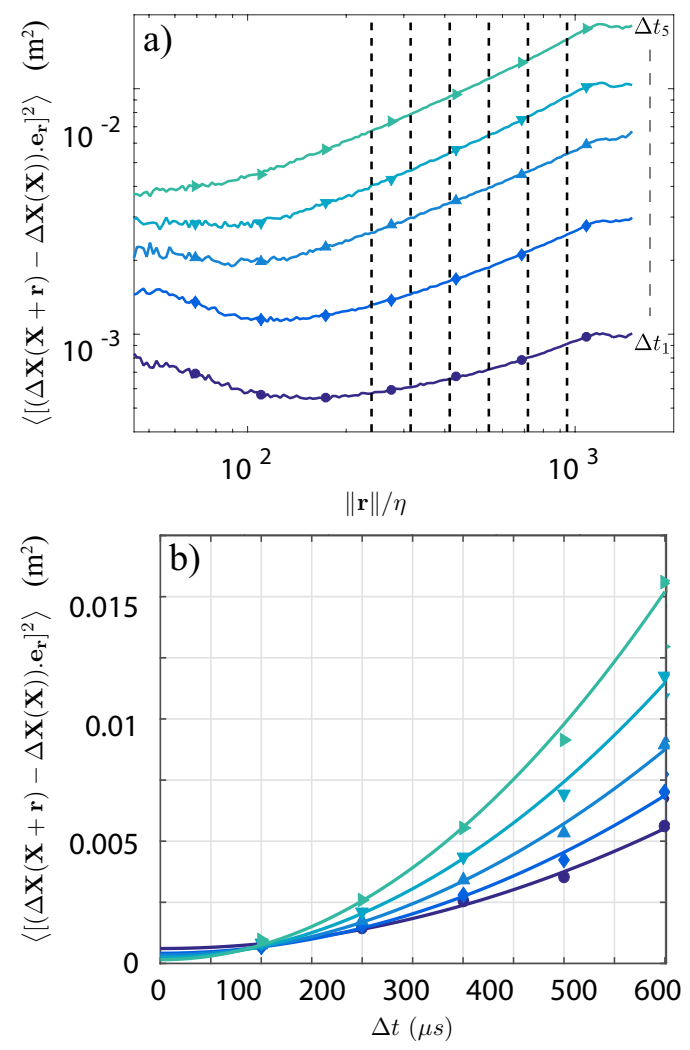

Figure 1. a) Longitudinal second order structure functions of the raw displacement field $\Delta \mathbf{X}$ against the separation $|\mathbf{r}|$ normalized by the Kolmogorov length scale $\eta$ for different values of $\Delta t_{i}$ equally-spaced from 0.05 to $0.2 \tau_{\eta}$ at $R e_{\lambda}=291$. b) Same quantities but plotted at a given separation $|\mathbf{r}|$ (indicated by the vertical dashed lines on (a); ascending order is for different values of $\Delta t$ ), as a function of the inter-frame time-step value $\Delta t$. The lines are fits of the form $c_{1} \Delta t^{2}+c_{2}$.

implementation of this denoising method. The plateaux found in the decade of separation values shown confirm the presence of the inertial range and their values correspond to the ensemble average of the local dissipation rate. The estimations of $\varepsilon$, as well as $u^{\prime}$ (spatial average of the fluctuating velocity map), for different Reynolds numbers compare well with those in [18], obtained by 2D3C PIV, confirming the accuracy of the method. In fact, the values of $u^{\prime}$ and $\varepsilon$ are slightly lower than those obtained by PIV. This discrepancy can be explained, qualitatively, based on the physics of the measurements and the effect of the noise on these metrics when it is not 

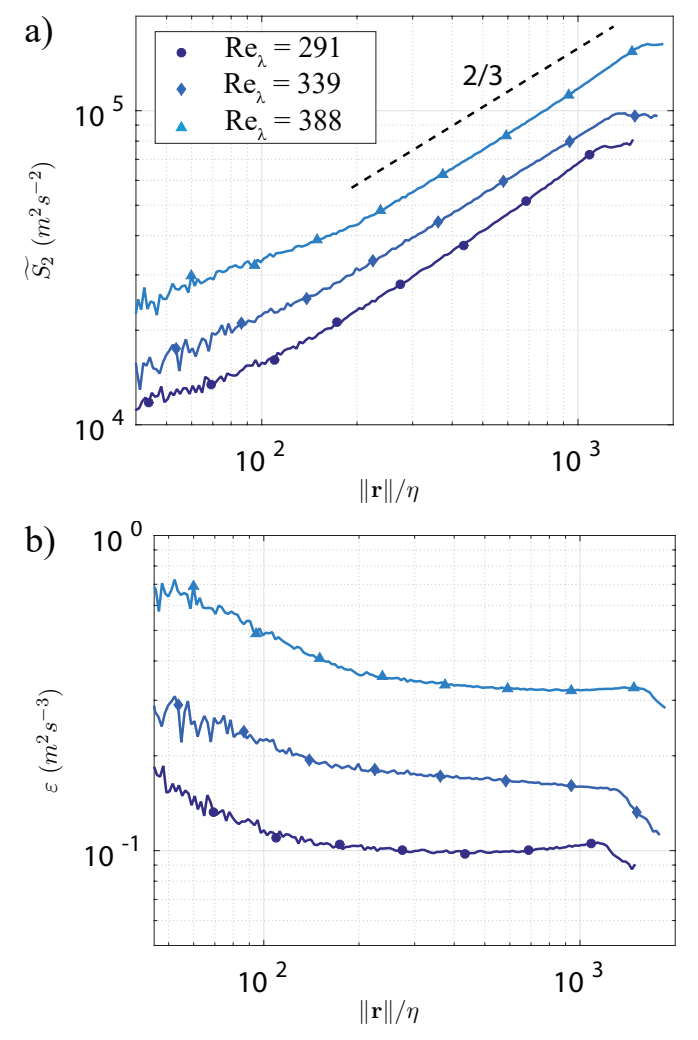

Figure 2. a) Second order structure functions of the velocity extracted with the proposed method for different $R e_{\lambda}$. The black dashed line corresponds to a power law of exponent 2/3. b) Energy dissipation rate estimated as $\varepsilon_{r}={\widetilde{S_{2}}}^{3 / 2} /|\mathbf{r}|$.

eliminated from the displacement measurements. Previous velocity measurements in the same experiment, conducted by traditional PIV [18], corresponded well with the actual velocity measured with this technique, but with noise variance convoluted onto it. The structure function (and hence $\varepsilon$ ) measured with traditional techniques was also subject to this erroneous increase in the value due to the creep-in of the noise into the computation of this statistical value. Equation 2 shows that the term $\left\langle\left[(\Delta \mathbf{b}(\mathbf{X}+\mathbf{r})-\Delta \mathbf{b}(\mathbf{X})) \cdot \mathbf{e}_{\mathbf{r}}\right]^{2}\right\rangle$ will increase the value $\varepsilon$ due to noise. To determine the importance of this term, it has to be expanded into $4\left\langle\mathbf{b}^{2}\right\rangle\left(1-C_{b}(|\mathbf{r}|)\right)$, where $C_{b}(|\mathbf{r}|)$ is the noise spatial correlation, bounded between $(-1,1)$. Regardless of the value of $C_{b}$, it will increase erroneously the value of the structure function yielding a higher value of $\varepsilon$ and because the value of $C_{b}$ depends on spatial separation, it will 
not raise it uniformly for all values of $|\mathbf{r}|$, changing the slope of the structure function with separation, thus making the value of $\varepsilon$ noisier.

\section{Discussion}

The comparison of the flow statistics with a previous 2D3C PIV study [18] allows for the validation of the proposed method. In fact, the measurements show better results, with no need to tune arbitrary filtering parameters to remove noise (the interrogation window size for instance). The only parameters that have to be chosen for the new method proposed here are the different values of $\Delta t$ that are accessible for a given flow and camera/illumination available, the form of the fit function and finally the binning in space to compute the Eulerian average and fluctuating velocities (if so desired), and in separation distance to compute the structure function.

The values of $\Delta t$ are subject to two limitations. They have to be high enough so that particles move more than the measurement error, while keeping the large displacements associated with highest $\Delta t$ from interfering with the ability of the particle tracking algorithm to identify individual particles [20]. As mentioned in the previous section, a maximum value of $\Delta t \lesssim \tau_{\eta}$ ensures that the third order correction (section 2) remains small $\left(u^{\prime 2} /\langle\widetilde{\mathbf{a}} \widetilde{\mathbf{v}}\rangle_{t} \tau_{\eta} \sim R e_{\lambda}\right)$. This was verified in the present experimental set-up and we found this correction to be negligible compared to the second order term. This was also the case for the structure function provided the separation lies in the inertial range $|\mathbf{r}| \gg \eta$. In such cases, the best agreement between fit functions and the data overall was found when using a quadratic function of $\Delta t$. As for the number of time-step values needed, the value of $\varepsilon$ when using only the three larger values of $\Delta t$ was only $5 \%$ lower than when using all five datasets. Using only the lowest value and largest values of $\Delta t$ allowed for a simple calculation of $\varepsilon$ that was only $2 \%$ higher than with the full experimental set.

The displacement vector field obtained from Particle Tracking in this multitime-step method is computed in a Lagrangian frame of reference. To compute the values of $\left\langle(\Delta \mathbf{X})^{2}\right\rangle_{t}$ against $\Delta t$, the displacement field must be binned into a spatial grid, converting it to an Eulerian frame of reference. Although the number of particles per image, or Eulerian grid cell, is relatively small in these PTV images, the velocity is estimated independently for each particle pair. Thus, the statistical convergence in the method is reached relatively quickly (without the need for a very large number of image pairs). The computation of the structure functions highlights 
this advantage. As pointed out above (section 3), the structure function could in principle be computed to arbitrarily small separation between particles. However, great care has to be taken in doing so because: i) difficulty to achieve statistical convergence in finding particles with small separations. ii) the second and third order terms in equation 2 will become of the same order of magnitude as the separation enters the dissipation range $(|\mathbf{r}| \sim 10 \eta)$. These explain why an increase of the structure functions at small separations is observed in figure 1a.

This note presents a new method to remove measurement noise from the calculation of velocity statistics, without filtering. It has been tested to compute the value of the energy dissipation rate through the second order structure function of the velocity in an experiment imaging tracer particles in a turbulent flow via stereoscopic particle tracking. The flow statistics computed are in good agreement with a previous 2D3C PIV study, with improved better accuracy.

\section{Acknowledgments}

The authors would like to acknowledge the financial support of the EUHIT project (no: 312778), Labex TEC 21 (ANR-11-LABX-0030), ENS de Lyon, ANR-12-BS090011, and PALSE/2013/26.

\section{References}

[1] R. J. Adrian, Annual review of fluid mechanics 23, 261 (1991)..

[2] Y. Sato, and K. Yamamoto, Journal of Fluid Mechanics, 175, 183-199. (1987).

[3] M. Virant, and T. Dracos, Measurement Science and Technology, 8(12), 15391552. (1997).

[4] G. A. Voth, A. L. Porta, A. Crawford, J. Alexander, and E. Bodenschatz, Journal of Fluid Mechanics 469, 121 (2002).

[5] Springer Handbook of Experimental Fluid Dynamics, C. Tropea, A. Yarin, and J. Foss, pp. 789-799 (Springer-Verlag, Berlin, 2007).

[6] H. Xu, Measurement Science and Technology 19(7), 075105 (2008).

[7] N. T. Ouellette, H. Xu, and E. Bodenschatz, Experiments in Fluids 40(2), 301-313 (2006).

[8] C. Cierpka, B. Lütke, and C. J. Kähler, Experiments in Fluids, 54(5), 1533 (2013).

[9] D. Schanz, S. Gesemann, and A. Schröder, Experiments in fluids, 57(5), 1-27 (2016).

[10] N. T. Ouellette, Probing the statistical structure of turbulence with measurements of tracer particle tracks (2006).

[11] S. Scharnowski, and C. J. Kähler, Experiments in Fluids, 57(2), 1-11 (2016). 
[12] N. Machicoane, M. Lopez-Caballero, M. Bourgoin, A. Aliseda, and R. Volk, arXiv preprint arXiv:1605.03803 (2016).

[13] F. Toschi and E. Bodenschatz, Annual Review of Fluid Mechanics, 41, 375 (2009).

[14] N. Mordant, A. Crawford, and E. Bodenschatz, Physica D, 193, (2004).

[15] R. Volk, N. Mordant, G. Verhille, and J.-F. Pinton, European Physics Letters, 81, 34002 (2008).

[16] J. Berg, S. Ott, J. Mann, and B. Luthi, Physical Review E, 80(2), (2009).

[17] R.Zimmermann, H. Xu, Y. Gasteuil, M. Bourgoin, R. V. ans J.-F. Pinton, and E. Bodenschatz, Rev. of Sci. Instrum, 81 (2010).

[18] L. Fiabane, R. Zimmermann, R. Volk, J.-F. Pinton, and M. Bourgoin, Phys. Rev. E 86 (2012).

[19] A. N. Kolmogorov, in Dokl. Akad. Nauk SSSR (1941), 30, 299-303.

[20] N. T. Ouellette, H. Xu, M. Bourgoin, and E. Bodenschatz, New Journal of Physics, 8, 109 (2006). 